\title{
Guided parent-delivered cognitive behaviour therapy for children with anxiety disorders: Outcomes at 3- to 5-year follow-up
}

\author{
Alexandra Brown 'D, Cathy Creswell ${ }^{2} *$, Chris Barker', Stephen \\ Butler', Peter Cooper ${ }^{2}$, Catherine Hobbs ${ }^{3}$ and Kerstin Thirlwall ${ }^{2}$ \\ 'University College London, UK \\ ${ }^{2}$ University of Reading, UK \\ ${ }^{3}$ University of Bath, UK
}

Objectives. Brief guided parent-delivered cognitive behaviour therapy (CBT) has been developed to meet the demand for non-intensive interventions for children with anxiety disorders, and initial trials have shown it to be effective for children with a range of anxiety disorders. This study examined outcomes $3-5$ years post-treatment.

Design. A long-term follow-up (LTFU) cohort study.

Methods. Families who (I) completed at least $50 \%$ of allocated treatment sessions of guided parent-delivered CBT for childhood anxiety as part of a randomized control trial (RCT), (2) provided consent to be recontacted, (3) had not received further mental health interventions, and (4) were contactable were invited to take part. Fifty-seven families (29\% of the original sample) completed structured diagnostic interviews on average 50 months after treatment (39-6I months).

Results. At LTFU, $79 \%$ of the assessed children who had received the treatment no longer met criteria for their primary diagnosis, $63 \%$ did not meet criteria for any anxiety disorder, and $61 \%$ did not meet criteria for any DSM-IV disorder. Treatment gains were mostly maintained $(60 \%)$, and some children went on to recover during the follow-up period without additional input from mental health services (19\%). Few young people had relapsed since their last assessment (I2\%). Mean scores on standardized symptom questionnaires were within the normal range.

Conclusions. Children who recovered from anxiety disorders following brief guided parent-delivered CBT typically maintained good outcomes and few relapsed. These findings suggest that this is a viable first-line, low-intensity treatment approach. This study only included a small subsample of those in the original RCT (29\%), and more information is required about those who dropped out of treatment and those who required further intervention immediately after treatment.

*Correspondence should be addressed to Cathy Creswell, School of Psychology and Clinical Language Sciences, University of Reading, Whiteknights, PO Box 2 I 7, Reading, Berkshire RG6 6AH, UK (email: c.creswell@reading.ac.uk). 


\section{Practitioner points}

- Treatment gains from brief guided parent-delivered cognitive behaviour therapy for children with anxiety are maintained for most children 3-5 years later.

- The majority of children who completed at least $50 \%$ of the intervention required no further mental health intervention in that time.

- Some children make continued improvement after completing the intervention.

- Data are based on a sample of families from southern England where the primary caregiving parent was free of mental health difficulties.

- Further research is needed to explore the mental health needs of those who do not benefit from this intervention.

Childhood anxiety disorders are common, affecting $6.5 \%$ of children worldwide (Polanczyk, Salum, Sugaya, Caye, \& Rohde, 2015). Cognitive behaviour therapy (CBT) is an effective treatment (Cartwright-Hatton, Roberts, Chitsabesan, Fothergill, \& Harrington, 2004; Compton et al., 2014; James, James, Cowdrey, Soler, \& Choke, 2013); however, it typically requires specialist resources and is often not available to those who might benefit from it (Stallard, Udwin, Goddard, \& Hibbert, 2007).

To meet the demand for effective, evidence-based treatment, brief approaches that use less face-to-face contact with a therapist than traditional approaches have been developed and evaluated. These have included computerized CBT (e.g., Spence et al., 2011) and guided parent-delivered CBT for parents (GPD-CBT; e.g., Lyneham \& Rapee, 2006; Thirlwall et al., 2013). These approaches can be used within a stepped-care model, where low-intensity interventions are offered for mild-to-moderate difficulties and high-intensity interventions are reserved for clients with more severe problems or those who do not respond to low-intensity treatment (Bower \& Gilbody, 2005).

Initial trials of GPD-CBT for anxious children have shown that this approach can bring about rates of recovery comparable to CBT delivered to children themselves (Cobham, 2012; Leong, Cobham, de Groot, \& McDermott, 2009). Thirlwall et al. (2013) evaluated two brief forms of this type of treatment in the UK health care system. After eight sessions of GPD-CBT (approximately $5 \mathrm{hr}$ of therapist contact), 50\% of children with anxiety disorders recovered from their primary anxiety disorder, and this was significantly better than the outcome of those in a waitlist control group. Children whose parents received four sessions of GPD-CBT (approximately $2.5 \mathrm{hr}$ of therapist contact) did not show superior outcomes compared to waitlist at post-treatment. Notably, at 6-month follow-up all children were found to have continued to make gains, irrespective of treatment intensity, with over $70 \%$ in both treatment groups being free of their primary disorder. Despite these promising findings, no studies have examined the longer term prognosis of children treated with this approach and, in particular, whether treatment gains are maintained over time following this brief intervention.

\section{Aims}

This study examined whether outcomes achieved using GPD-CBT are maintained at 3- to 5-year follow-up, without further input from mental health services.

\section{Method}

\section{Participants}

Participants were families who took part in a randomized controlled trial of brief GPD-CBT (Thirlwall et al., 2013). The original trial included follow-up assessments post-treatment 
and 6 months later. They were invited to join the present follow-up study if they had completed at least $50 \%$ of the treatment sessions and had given consent to be contacted again. Families were excluded if the child had received any further treatment for a mental health problem so that only treatment effects of the GPD-CBT intervention were being measured.

A total of 194 families participated in the 2013 trial. One hundred and fifty families had completed at least $50 \%$ of the treatment, and all of these had given consent at the time of treatment to be contacted about a further follow-up study. These 150 families were therefore contacted and invited to participate. Of these, $30(20 \%)$ declined to participate, $33(22 \%)$ were excluded as the child had received further treatment for a mental health problem, and 22 (15\%) could not be reached by telephone, email, or letter (see Figure 1). Of those 33 who had received further treatment, 17 had further treatment for anxiety, two had medication for Attention Deficit Hyperactivity Disorder, two had subsequently been diagnosed with Autistic Spectrum Disorder, two had developed eating disorders, and 10 had other unspecified conditions for which they sought treatment.

Thus, 65 of the original sample of 150 families (43\%) participated in the follow-up assessments. Fifty-seven families were interviewed using the ADIS-C/P and completed questionnaires (48 using both child and parent interviews; nine families had parent interviews only). Eight further families completed questionnaires only.

\section{Measures}

The primary and secondary outcome measures used at post-treatment and 6-month follow-up were repeated.

\section{Primary outcome measures}

Anxiety Disorders Interview Schedule for DSM-IV: Child and parent versions (ADIS-C/P; Silverman EAlbano, 1996). This semi-structured interview for both child and parent primarily assesses anxiety disorders according to DSM-IV diagnostic criteria, but also gathers data on mood, externalizing behaviour, and other mental health problems. Assessments were carried out by one of two assessors face-to-face or over the telephone. For each assessor, the first 20 assessments were discussed with an experienced diagnostician (postgraduate psychologist with extensive training and experience with the ADIS-C/P). The assessor and diagnostician both generated independent ratings on the basis of the discussion with reference to audio recordings as required. The assessor was deemed to be reliable if inter-rater reliability for both diagnoses and clinician severity ratings (CSRs) between the assessor and consensus assessor exceeded .85. Every sixth assessment thereafter was discussed and doublecoded with the consensus assessor to prevent rater drift. Both assessors achieved kappa levels in excess of .95 for diagnostic classifications and intraclass correlations of over .95 for CSRs.

Clinical Global Impression-Improvement Scale (CGI-I; Guy, 1976). The CGI-I is a 7-point clinician-rated scale measuring the child's improvement from baseline, where lower scores indicate greatest improvement. As with the ADIS-C/P, the first 20 ratings were discussed with an experienced rater and reliability was formally assessed. Both assessors achieved kappa levels in excess of .85. 


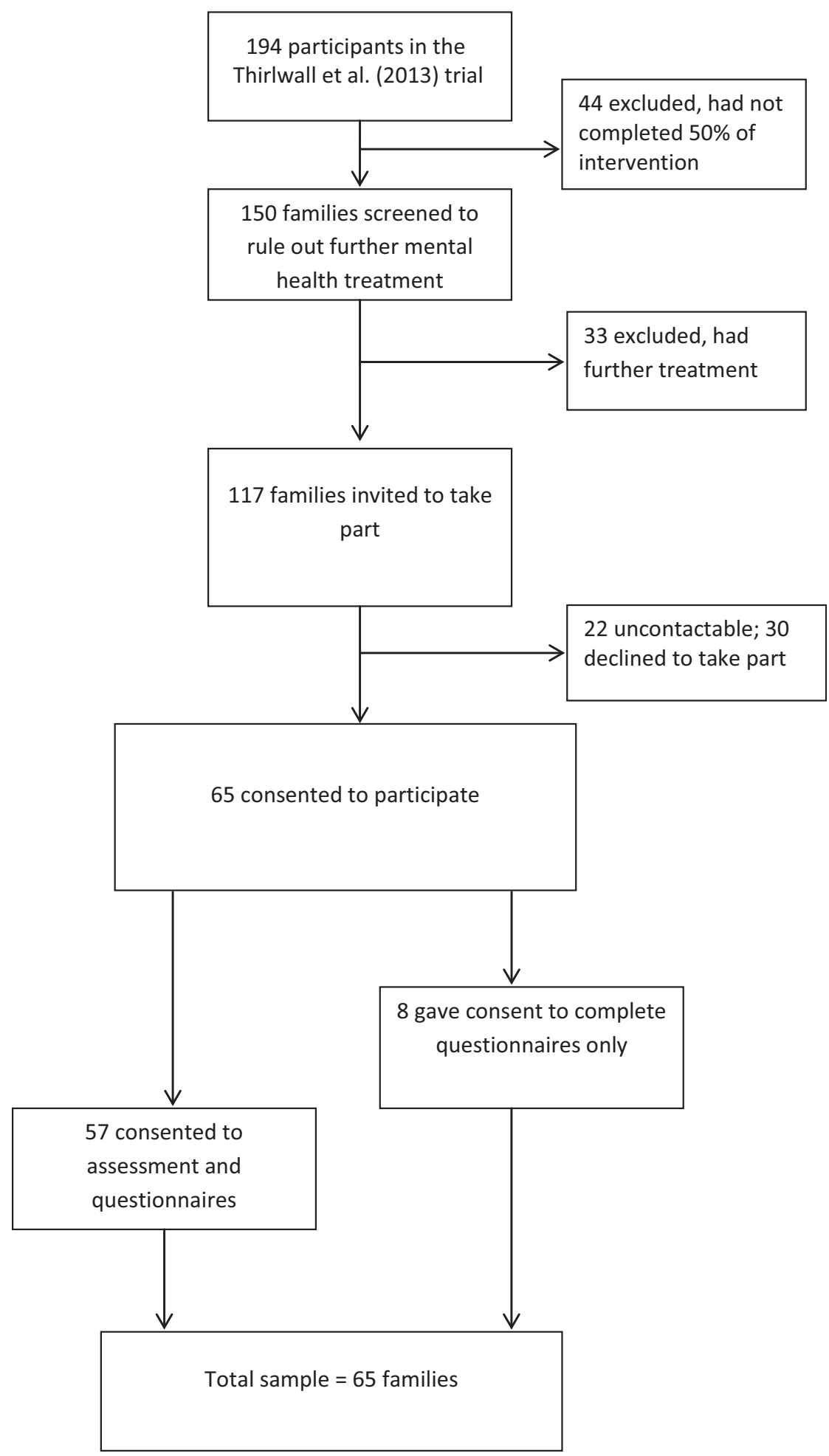

Figure I. Participant flow, withdrawals, and exclusions. 
Secondary outcome measures

Spence Children's Anxiety Scales: Child and parent versions (SCAS-C/P; Spence, 1998). The SCAS-C/P is a 44-item scale rating anxiety symptom severity, in line with DSM-IV diagnostic criteria. Cronbach's alpha was .92 for child report and .91 for parent report.

Child's Anxiety Impact Scale: Child and parent versions (CAIS-C/P; Langley, Bergman, McCracken, E Piacentini, 2004). Parents and children rate 34 items to indicate the impact of anxiety on functioning in home, family, and social domains. Cronbach's alpha was .83 for child report and .86 for parent report.

Short Mood and Feelings Questionnaire: Child and parent versions (SMFQ-C/P; Angold, Costello, \& Messer, 1995). The SMFQ-C/P is a 13-item questionnaire measuring symptoms of depression, corresponding to DSM-IV diagnostic criteria. Cronbach's alpha was .89 for child report and .74 for parent report.

Strengths and Difficulties Questionnaire (Goodman, 1997). The Strengths and Difficulties Questionnaire (SDQ) is a 25-item behavioural checklist measuring emotional symptoms, conduct problems, hyperactivity, peer relationship problems, and pro-social behaviour. Only the conduct problems scale was used in this study. Cronbach's alpha was .64 for child report and .41 for parent report on the conduct problems scale.

\section{Intervention}

In the original trial, parents were given a self-help book ('Overcoming Your Child's Fears and Worries'; Creswell \& Willetts, 2007) and received one of two forms of therapist support: 'full' support (i.e., 4 hr-long face-to-face sessions and four 20-min telephone contacts) or 'brief' support (i.e., 2 hr-long face-to-face sessions and two 20-min telephone contacts) in working through the programme. The programme followed a CBT approach, which included identifying and testing thoughts, graded exposure, and problem-solving. Parents completed homework tasks between sessions.

\section{Results}

\section{Sample characteristics}

Long-term follow-up (LTFU) assessments took place 39-61 months after the initial assessment, with a mean follow-up period of 50 months ( $S D=6.2$ months). Young people were aged 11-17 years at follow-up. The LTFU sample was compared to the remainder of the overall sample on key baseline variables. The two groups did not differ significantly on mother-reported SDQ-conduct, $F(1,180)=0.89, p=.348$, childreported SMFQ scores, $F(1,184)=2.13, p=.146$, or the frequency of one or more comorbid anxiety diagnosis, $\chi^{2}(1, N=194)=1.71, p=.191$. However, the LTFU group were younger, $F(1,162)=8.92, p=.003$ (LTFU mean $=9$ years 0 months; non-LTFU mean $=9$ years 9 months at initial assessment), and had lower scores on motherreported SCAS pre-treatment, $F(1,162)=7.94, p=.005$. 


\section{Diagnostic status}

Diagnostic data from ADIS assessments at LTFU were available for 57 participants. Participants were categorized according to whether they met diagnostic criteria for (1) their pre-treatment primary diagnosis, (2) any anxiety disorder, and (3) any anxiety, mood, or behaviour disorder. The majority of assessed children, all of whom met strict diagnostic criteria for an anxiety disorder before treatment, no longer met criteria for their primary diagnosis (79\%) and were rated as 'much/very much improved' on the CGI-I (79\%). Frequencies at all time points are shown in Table 1.

\section{Change in diagnostic status}

Diagnostic status at LTFU was compared to diagnostic status at the last available assessment for that participant: 11 participants (19\%) who had met criteria at their last assessment had since recovered, 34 (60\%) had recovered at their last assessment and remained so, 7 (12\%) had recovered at their last assessment and had since relapsed, and 5 (9\%) had met diagnostic criteria for an anxiety disorder at their last assessment and continued to do so at LTFU.

\section{Secondary outcome measures}

Parent-reported SCAS, CAIS, SMFQ, and SDQ-C total scores were available for 63 participants. Child self-report scores were available for 54 of these. Means and standard deviations for all time points are reported in Table 2. Mean scores on the SCAS and CAIS questionnaires at LTFU were comparable to those found in normative samples (Langley et al., 2004; Nauta et al., 2004). Mean scores on the SMFQ fell below the clinical cut-off of 11 (Angold, Erkanli, Silberg, Eaves, \& Costello, 2002), and scores on the SDQ-C fell within the normative range (Goodman, 2001).

\section{Discussion}

Bower and Gilbody (2005) specify that the first recommended treatment within a steppedcare model should be 'the least restrictive of those available, but still likely to provide significant health gain' (p. 11). Brief GPD-CBT is less restrictive than traditional CBT for childhood anxiety disorders in terms of the time required for both therapists and families. The findings of the current study suggest that this mode of treatment delivery is likely to provide significant long-term health gain for some children with anxiety disorders. For those who completed the treatment and received no further intervention - who were the focus of the current study - recovery was most commonly maintained or achieved in the approximately 3-5 years following treatment. Few children who had recovered were found to have relapsed at follow-up. At the LTFU, mean scores on measures of anxiety symptoms, anxiety interference, low mood, and externalizing behaviour symptoms were within the 'normal' range. These findings are consistent with studies from more traditional CBT formats, which often show that treatment gains are maintained several years after treatment ends (Barrett, Duffy, Dadds, \& Rapee, 2001; Kendall, Safford, Flannery-Schroeder, \& Webb, 2004; Saavedra, Silverman, Morgan-Lopez, \& Kurtines, 2010).

Strengths of the study include the use of semi-structured diagnostic interviews to enable comprehensive assessments of mental health and diagnostic criteria to determine 


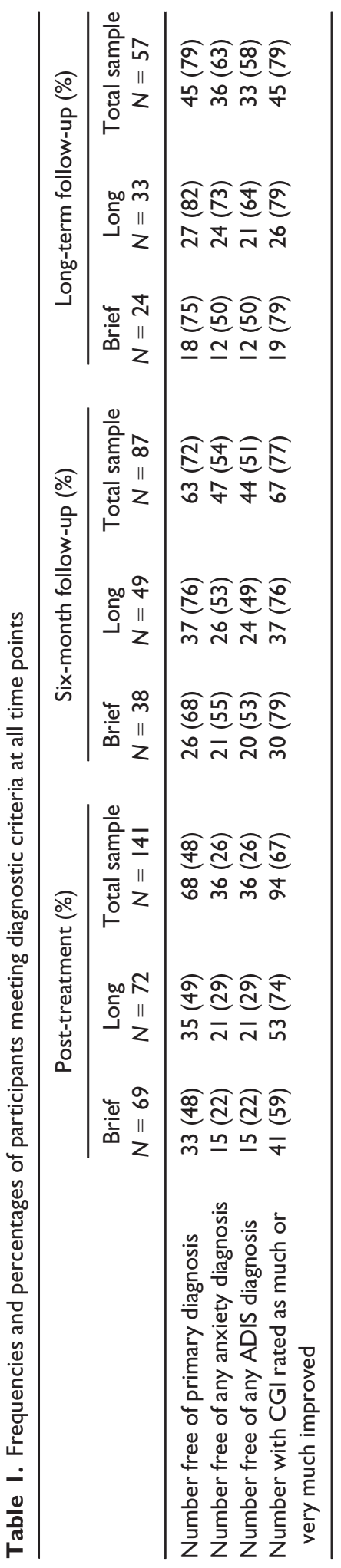




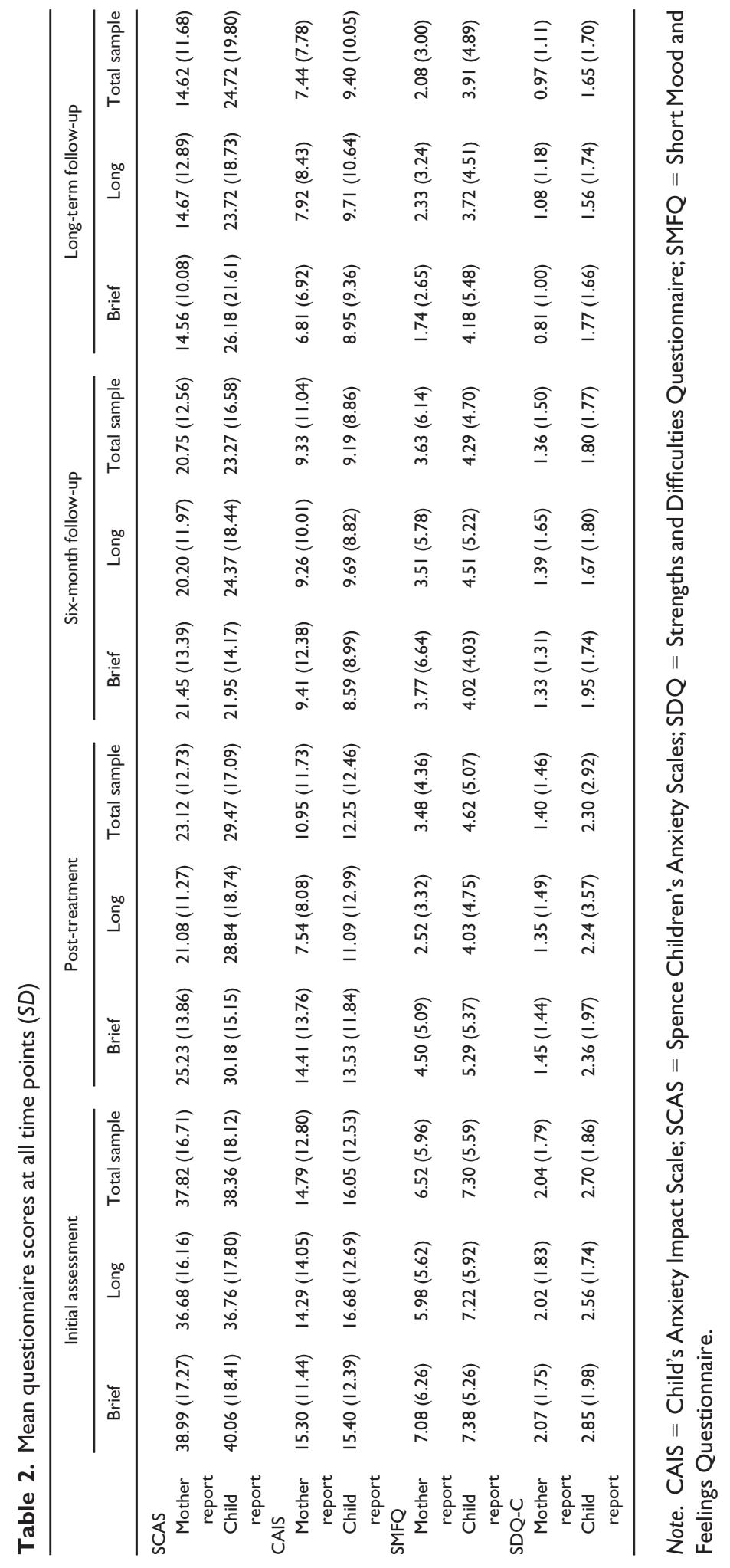


improvement and recovery. All of the LTFU assessments and clinician ratings were conducted by independent assessors, not involved in the original trial and unaware of previous clinical status. Scores on both self-report and parent-report symptom measures converged with clinician ratings and diagnostic data, although only parent-reported data were available for some participants.

A number of important limitations should also be noted. Although it is known that childhood anxiety disorders commonly run a chronic course (Essau, Conradt, \& Petermann, 2002), the lack of a comparison group means that we cannot be certain that improvements were not a result of naturalistic recovery over time. Furthermore, 44 (23\%) participants from the original trial were excluded from this follow-up as they had not completed at least half of the treatment sessions offered, suggesting that the intervention may not have been sufficient for these young people. We also combined participants who had received a $2.5-\mathrm{hr}$ and a 5 -hr version of GPD-CBT on the basis that outcomes were very similar by the 6-month follow-up assessment; however, the initial trial was not powered to establish either superiority or equivalence of the two treatment formats.

A key limitation of this study is attrition from the original sample. Only $56 \%$ of eligible participants took part, with the result that only 34\% of participants who entered the original trial provided questionnaire measures or a structured interview. Long-term outcomes for most participants therefore remain unknown. As we were keen to establish outcomes for those who had not received further treatment, we did not invite the $22 \%$ of eligible children who went on to have further intervention to this study. As such, conclusions are limited to those who both completed treatment and required no further treatment after this low-intensity treatment. Future studies are required which evaluate outcomes over the full course of a stepped-care approach to treatment. A significant proportion of children were also unwilling to take part, or not contactable (35\%), highlighting the difficulty associated with conducting follow-up research after an extended period, even when participants were informed about the possibility of further research at the outset of the original trial.

A further limitation is the incomplete data on the participants who went on to seek further mental health treatment. It remains unknown whether these young people were referred for further treatment because of treatment failure, or whether they had recovered from their primary diagnosis and then relapsed, or sought help for a comorbid diagnosis. Indeed, in some cases families specified that treatment was for other conditions (such as autistic spectrum conditions and eating disorders). The nature of ongoing mental health needs in children who do not respond to low-intensity treatment for anxiety warrants further study.

The sample used in our original trial (Thirlwall et al., 2013) trial was drawn from a relatively affluent, educated, predominantly White, British population where most households included two parents. Furthermore, families where the main caregiving parent was currently suffering from an anxiety or mood disorder were excluded from the trial. This resulted in a sample with a relatively good prognosis and perhaps optimal characteristics to make use of a low-intensity intervention using written materials, which may not be representative of all clinical populations.

The measure that was used to examine behaviour problems, the conduct problems scale of the SDQ, showed low internal consistency, particularly for parent-reported behaviour difficulties. This may be due to the scale comprising only five items which ask about both common behaviour difficulties which most children may show to some degree (i.e., 'hot tempers' and disobedience) and less common but more serious behaviour 
problems (i.e., stealing, fighting) which were rarely seen in this sample. Several other studies have reported similarly low internal consistency for the conduct problems scale (e.g., Rønning, Handegaard, Sourander, \& Mørch, 2004), and as such, the findings should be interpreted with caution.

\section{Conclusions}

Findings from this study suggest that children whose parents complete GPD-CBT and are not immediately stepped up to further treatment typically maintain good outcomes. Further research should focus on identifying which children GPD-CBT is most appropriate for, which children will require more intensive input, and what this should comprise.

\section{References}

Angold, A., Costello, E., \& Messer, S. (1995). Development of a short questionnaire for use in epidemiological studies of depression in children and adolescents. International Journal of Methods in Psychiatric Research, 5, 237-249.

Angold, A., Erkanli, A., Silberg, J., Eaves, L., \& Costello, E. J. (2002). Depression scale scores in 8-17 year-olds: Effects of age and gender. Journal of Child Psychology and Psychiatry, 43, 1052-1063. doi:10.1111/1469-7610.00232

Barrett, P. M., Duffy, A. L., Dadds, M. R., \& Rapee, R. M. (2001). Cognitive-behavioural treatment of anxiety disorders in children: Long-term (6-year) follow-up. Journal of Consulting and Clinical Psychology, 69, 135-141. doi:10.1037//0022-006x.69.1.135

Bower, P., \& Gilbody, S. (2005). Stepped care in psychological therapies: Access, effectiveness and efficiency. Narrative literature review. The British Journal of Psychiatry, 186, 11-17. doi:10. 1192/bjp.186.1.11

Cartwright-Hatton, S., Roberts, C., Chitsabesan, P., Fothergill, C., \& Harrington, R. (2004). Systematic review of the efficacy of cognitive behaviour therapies for childhood and adolescent anxiety disorders. The British Journal of Clinical Psychology, 43, 421-436. doi:10.1348/ 0144665042388928

Cobham, V. E. (2012). Do anxiety-disordered children need to come into the clinic for efficacious treatment? Journal of Consulting and Clinical Psychology, 80, 465-476. doi:10.1037/ a0028205

Compton, S. N., Peris, T. S., Almirall, D., Birmaher, B., Sherrill, J., Kendall, P. C., .. Albano, A. M. (2014). Predictors and moderators of treatment response in childhood anxiety disorders: Results from the CAMS trial.Journal of Consulting and Clinical Psychology, 82, 212-224. doi:10.1037/ a0035458

Creswell, C., \& Willetts, L. (2007). Overcoming your child's fears and worries: A self-help guide using cognitive-behavioural techniques. London, UK: Constable Robinson.

Essau, C. A., Conradt, J., \& Petermann, F. (2002). Course and outcome of anxiety disorders in adolescents. Journal of Anxiety Disorders, 16, 67-81. doi:10.1016/s0887-6185(01)00091-3

Goodman, R. (1997). The strengths and difficulties questionnaire: A research note. Journal of Child Psychology and Psychiatry, 38, 581-586. doi:10.1111/j.1469-7610.1997.tb01545.x

Goodman, R. (2001). Psychometric properties of the Strengths and Difficulties Questionnaire (SDQ). Journal of the American Academy of Child and Adolescent Psychiatry, 40, 1337-1345. doi:10.1097/00004583-200111000-00015

Guy, W. (1976). The Clinical Global Impression Scale, ECDEU assessment manual for psychopharmacology. Rockville, MD: USA Department of Health, Welfare and Public Health Services. doi:10.1037/e591322011-001 
James, A. C., James, G., Cowdrey, F. A., Soler, A., \& Choke, A. (2013). Cognitive behavioural therapy for anxiety disorders in children and adolescents. The Cochrane Database of Systematic Reviews, 6, CD004690. doi:10.1002/14651858.cd004690.pub3

Kendall, P. C., Safford, S., Flannery-Schroeder, E., \& Webb, A. (2004). Child anxiety treatment: Outcomes in adolescence and impact on substance use and depression at 7.4-year follow-up. Journal of Consulting and Clinical Psychology, 72, 276-287. doi:10.1037/0022-006x.72.2.276

Langley, A. K., Bergman, R. L., McCracken, J., \& Piacentini, J. C. (2004). Impairment in childhood anxiety disorders: Preliminary examination of the child anxiety impact scale-parent version. Journal of Child and Adolescent Psychopharmacology, 14, 105-114. doi:10.1089/ 104454604773840544

Leong, J., Cobham, V. E., de Groot, J., \& McDermott, B. (2009). Comparing different modes of delivery: A pilot evaluation of a family-focused, cognitive-behavioral intervention for anxietydisordered children. European Child \& Adolescent Psychiatry, 18, 231-239. doi:10.1007/ s00787-008-0723-7

Lyneham, H. J., \& Rapee, R. M. (2006). Evaluation of therapist-supported parent-implemented CBT for anxiety disorders in rural children. Behaviour Research and Therapy, 44, 1287-1300. doi:10.1016/j.brat.2005.09.009

Nauta, M. H., Scholing, A., Rapee, R. M., Abbott, M., Spence, S. H., \& Waters, A. (2004). A parentreport measure of children's anxiety: Psychometric properties and comparison with childreport in a clinic and normal sample. Behaviour Research and Therapy, 42, 813-839. doi:10. 1016/s0005-7967(03)00200-6

Polanczyk, G. V., Salum, G. A., Sugaya, L. S., Caye, A., \& Rohde, L. A. (2015). Annual research review: A meta-analysis of the worldwide prevalence of mental disorders in children and adolescents. Journal of Child Psychology and Psychiatry, 56, 345-365. doi:10.1111/jcpp.12381

Rønning, J. A., Handegaard, B. H., Sourander, A., \& Mørch, W. (2004). The Strengths and Difficulties Self-Report Questionnaire as a screening instrument in Norwegian community samples. European Child and Adolescent Psychiatry, 13, 73-82. doi:10.1007/s00787-004-0356-4

Saavedra, L. M., Silverman, W. K., Morgan-Lopez, A. A., \& Kurtines, W. M. (2010). Cognitive behavioral treatment for childhood anxiety disorders: Long-term effects on anxiety and secondary disorders in young adulthood. Journal of Child Psychology and Psychiatry, and Allied Disciplines, 51, 924-934. doi:10.1111/j.1469-7610.2010.02242.x

Silverman, W. K., \& Albano, A. M. (1996). The Anxiety Disorders Interview Schedule for DSM-IVChild and parent versions. San Antonio, TX: Psychological Corporation.

Spence, S. H. (1998). A measure of anxiety symptoms among children. Behaviour Research and Therapy, 36, 545-566. doi:10.1016/s0005-7967(98)00034-5

Spence, S. H., Donovan, C. L., March, S., Gamble, A., Anderson, R. E., Prosser, S., \& Kenardy, J. (2011). A randomized controlled trial of online versus clinic-based CBT for adolescent anxiety. Journal of Consulting and Clinical Psychology, 79, 629-642. doi:10.1037/a0024512

Stallard, P., Udwin, O., Goddard, M., \& Hibbert, S. (2007). The availability of cognitive behaviour therapy within specialist child and adolescent mental health services (CAMHS): A national survey. Behavioural and Cognitive Psychotherapy, 35, 501-505. doi:10.1017/ s1352465807003724

Thirlwall, K., Cooper, P. J., Karalus, J., Voysey, M., Willetts, L., \& Creswell, C. (2013). Treatment of child anxiety disorders via guided parent-delivered cognitive-behavioural therapy: Randomised controlled trial. The British Journal of Psychiatry, 203, 401-402. doi:10.1192/bjp.bp.113. 129635

Received 9 June 2016; revised version received 30 November 2016 